\title{
Prostaglandin Analogs in the Cataract Surgery Perioperative Period
}

\author{
${ }^{1}$ Anurag Shrivastava, ${ }^{2}$ Kuldev Singh \\ ${ }^{1}$ Albert Einstein College of Medicine, Montefiore Medical Center, Bornx, New York, USA \\ ${ }^{2}$ Stanford University, Department of Ophthalmology, Palo Alto, California, USA
}

\section{INTRODUCTION}

Since being introduced over a decade ago, the prostaglandin analog class of medications has revolutionized medical therapy for the glaucoma patient. These agents are commonly used for initial glaucoma therapy in areas of the world where they are readily available and affordable. The popularity of this class stems from several attributes including a high level of effectiveness in lowering IOP, convenient dosing, and predominantly harmless ocular and systemic side effects in most patients. One exception with regard to side effects has been the fear that these medications are contraindicated in special situations. Specifically, many case reports and retrospective analyses have implicated the prostaglandin analog class of medications as being linked to the development of postoperative cystoid macular edema (CME) in patients who have undergone cataract surgery. While some have postulated that this is a drug related side effect, a few clinical studies have shown that it is the preservative used with the medication, rather than the drug itself, which is the root cause of CME. ${ }^{1}$ Yet another confounding factor is the variability of criteria for defining significant CME in the studies that have been reported. ${ }^{2}$ As the prevalence of ocular hypertension and glaucoma in patients undergoing cataract extraction is high, almost all cataract surgeons routinely face the question of whether or not it is safe to use prostaglandin analogs during the cataract surgery perioperative period. To date, there have been no randomized control trials providing Oxford Level I evidence demonstrating causality, and a preferred practice pattern has yet to be published on this subject. This lack of consensus was highlighted in the results of a Royal College of Ophthalmologists survey conducted by Ahad and McKee which demonstrated that approximately $60 \%$ of physicians did not stop prostaglandin analogs following uncomplicated cataract surgery. Of those who did stop treatment, approximately half did so routinely, while the other half did so only in patients deemed to be at high risk for postoperative CME. ${ }^{3}$ The purpose of this review is to summarize current available evidence with regard to this topic and to make recommendations for care based upon this evidence as well as the clinical experience of glaucoma experts.

\section{HOW PREVALENT IS CME FOLLOWING CATARACT EXTRACTION?}

While the prevalence of CME following cataract surgery would appear to be a relatively simple statistic to ascertain, the figure is largely dependant upon how one defines the condition. Clinical CME is characterized ophthalmoscopically as the presence of cystoids spaces in the macula, and has the lowest reported prevalence of all definitions. Clinically Significant $C M E$, on the other hand, refers to decreased Snellen visual acuity associated with angiographic findings signifying this condition. The prevalence of clinically significant CME has been reported to be between $1 \%$ and $5 \%{ }^{4,5}$ This relatively large range is predominantly due to a variety of predisposing conditions which dramatically influence the likelihood of postoperative CME. A third definition, Angiographic CME, simply refers to the characteristic angiographic findings, regardless of impact on vision, and has been reported to occur as commonly as $30 \%$ following extracapsular cataract extraction. ${ }^{6}$

When reviewing the literature on this topic, it is imperative that one not apply data from studies describing one type of CME to another definition utilizing a different criterion. From a study design perspective, angiographic CME is certainly an attractive end point, as the relatively high prevalence allows easier accumulation of evidence in support of or against causality. Additionally, this definition is the least subjective of the three mentioned above. On the other hand, clinical CME and clinically significant CME offer end points that are more relevant to patient outcomes relative to angiographic CME. Regardless of which endpoint is used in the definition, it is clear that a strict cause and effect relationship is difficult to establish given our rudimentary understanding of the disease process, along with the confounding factors commonly associated with the pathology of this condition.

\section{CASE REPORTS}

There are a plethora of reports in the literature that have attempted to establish a temporal association between the use 
of prostaglandin analogs and CME in pseudophakic patients. ${ }^{7-12}$ Generally speaking, these case reports attempt to associate the onset of CME with the initiation of prostaglandin therapy to reduce elevated intraocular pressure in the postoperative period. Furthermore, some such reports have demonstrated an impressive resolution of CME and prompt visual improvement upon the cessation of prostaglandin therapy. This resolution is usually associated with discontinuation of prostaglandin analog therapy in conjunction with the administration of topical steroidal and/or nonsteroidal anti-inflammatory medications (NSAIDs). ${ }^{8,9,11-15}$ The resolution of CME, if it is indeed associated with stopping prostaglandin analog therapy, with or without concomitant steroid or NSAID therapy, is an important practical consideration when it comes to caring for the post cataract surgery patient. Such reversibility of the process allows the ophthalmologist more flexibility when weighing the risks and benefits of continuation versus discontinuation of a prostaglandin analog following such surgery, taking into account an individual patient's risk of IOP related vision loss as well as other comorbidities. It is well recognized, however, that case reports and series are not appropriate substitutes for well-designed, adequately powered, randomized clinical trials, particularly when the potential impact of study findings on clinical care is significant.

\section{PATHOGENESIS OF CME AND WHO IS AT RISK?}

Based upon available CME incidence and prevalence data from patients followed after cataract surgery, it is generally accepted that the etiology of this process is multifactorial. Several studies have indicated that patients at high risk for CME include those with a history of retinal vein occlusion, diabetes, vitreous loss, uveitis, vitreomacular traction, epiretinal membrane, aphakia, and an absent posterior capsule (postcapsulotomy or via an intraoperative complication). ${ }^{14}$ Intuitively, the common denominator between these "high-risk" patients is vascular compromise at the intraretinal level. It is notable, however, that even within this group of patients with predisposing factors for $\mathrm{CME}$, the published rates of clinically significant CME are generally below $5 \% .^{9}$

Several modulators have been implicated in the causation of CME. These include complement, platelet-activating factor, cytokines, interleukin-6, lysosomal enzymes, vascular endothelial growth factor, and, most notably, products of the arachadonic acid cascade such as prostaglandins (PGE2, etc.). ${ }^{16}$ These mediators are thought to cause the breakdown of the blood-aqueous barrier and, ultimately, the blood-retina barrier, with both processes postulated to be contributing to the development of CME. Further, analysis via pharmacokinetic research has demonstrated, through the use of radiolabeled prostaglandin esters, that the level of topical medications in the retina and choroid is potentially high enough to affect retinal physiology. $^{16}$
It has been postulated that the initial insult leading to CME in pseudophakic patients is operative irritation of uveal tissue along with the release of lens epithelial cells. ${ }^{17}$ As the prostaglandin analog class of glaucoma medications is thought to work primarily through binding to the prostaglandin F receptor, some have postulated that such binding may trigger the process which leads to postoperative CME in patients using these medications. Another series of experiments, however, has demonstrated that the preservative used in prostaglandin analogs, rather than the medication itself, is the inciting factor in the pathogenesis of CME. The term "pseudophakic preservative maculopathy” (PPM), was coined to describe the finding that BAK-preserved solutions of both prostaglandins and timolol resulted in similar levels of inflammation relative to BAK preservative controls. ${ }^{1}$ On the other hand, a case of postoperative CME related temporally to Travatan Z, a BAKfree prostaglandin formulation, has also been reported. ${ }^{18}$ While the possibility that several different preservatives may play a role in this process cannot be excluded, conflicting findings on the topic suggest that a strict cause and effect relationship between prostaglandin analog useand postoperative CME has not as yet been established, and the issue of causality is clearly more complex than initially theorized.

\section{THE IMPORTANCE OF ANALYZING RISKS AND BENEFITS}

Given the lack of concordance between case reports and basic science research with regard to causality, it is difficult to establish routine preferred practice recommendations for the use of prostaglandin analogs in the cataract surgery perioperative period. The evidence suggests that one should be flexible in determining the use of these agents following cataract surgery with relative, rather than absolute, contraindications being appropriate in most circumstances. A detailed risk-benefit analysis for each patient is certainly justified. Notwithstanding the lack of proof that the perioperative use of prostaglandins causes $\mathrm{CME}$, it is reasonable to consider stopping these agents sometime prior to cataract surgery to minimize the potential risk. While the vast majority of patients do not have the aforementioned comorbities that place them at higher risk for CME, it is often in the patient's best interest to avoid postoperative complications if the benefits of treatment with prostaglandin analogs is out-weighed by the potential risk of CME. The practical question in this debate is not necessarily whether or not prostaglandin analogs and their associated preservatives can lead to CME. Rather, one must ask whether or not the risk of poorly controlled IOP for a relatively brief period in the life of an ocular hypertension or glaucoma patient outweigh the benefits of avoiding a relatively uncommon phenomenon that has not as yet been conclusively linked to a particular class of drugs? The requisite data set required to answer to this question is undoubtedly unique for each patient and thus decision making should be on a case by case basis. 


\section{DISCUSSION AND RECOMMENDATIONS}

One can make a good case that modern extracapsular cataract surgery, particularly when employing phacoemulsification, is the most commonly performed procedure for lowering IOP today. While there is great variability in what happens to the IOP among patients following cataract extraction with intraocular lens implantation, a drop in the average IOP has occurred after surgery in most study populations. ${ }^{19-21}$ Cataract surgery provides an excellent opportunity to stop glaucoma medications, adding them back only to the extent necessary in the postoperative period. In our clinical experience, a substantial proportion of patients who required prostaglandin therapy prior to cataract surgery will not need any glaucoma medications, even in the long-term postoperative period.

There is no Oxford Level I highest-quality evidence demonstrating harm from the use of prostaglandin analogs prior to or after cataract surgery. Furthermore, the current literature has failed to demonstrate a clear association between these drugs and CME or ocular inflammation. Preservatives used with multiple topical glaucoma medications including, but not limited to prostaglandin analogs, may be more significant than active drug components in instigating the cascade of events that lead to the development of CME if indeed there is an association. While further study of the causality issue with regard to prostaglandins and CME is clearly needed, the low incidence of the condition, even with the use of these agents in the perioperative period lead one to question whether precious research funds might not be better served answering other questions. Regardless of the actual cause and effect relationship between prostaglandin analogs and CME, common sense dictates the discontinuation of these medications in the immediate postoperative period, particularly in patients with preexisting risk factors that have been associated with an increased risk of this complication. Such decision-making is made easier by the fact that prostaglandin analogs are less than ideal for lowering IOP during the immediate postoperative period. Medications such as alpha adrenergic agonists, betablockers, and topical and systemic carbonic anhydrase inhibitors (CAIs), all of which decrease the production of aqueous humor, are generally faster acting than prostaglandin analogs and are not mediators of inflammation, making them desirable postoperative IOP-lowering agents. In contrast, prostaglandin analogs are well know mediators of inflammation, further casting doubt on the appropriateness of using these agents, particularly in the early postoperative period. Although conclusive evidence that topical ocular prostaglandin use causes or exacerbates intraocular inflammation is lacking, most ophthalmologists would agree that using these agents in the early postoperative period is not optimal.

In cases where intraocular pressure can be temporarily controlled with other medications including beta-blockers, alpha adrenergic agonists and topical or oral carbonic anhydrase inhibitors, we recommend discontinuation of prostaglandin analog therapy approximately 1 week prior to cataract extraction, particularly in eyes considered at high risk for the development of postoperative CME. Given that there is no evidence that such discontinuation decreases the likelihood of postoperative CME, the one-week time period is clearly arbitrary.

As the majority of IOP spikes after cataract extraction occur within the first postoperative week, commonly associate with retained viscoelastic combined with intraocular inflammation, we recommend that a prostaglandin analog should be the last pharmacologic choice for lowering IOP during this period for a variety of reasons. These transient IOP spikes can generally be controlled by aqueous suppressants such as alpha-adrenergic agonists, beta-blockers, or topical and oral CAIs, none of which have been postulated to cause intraocular inflammation. This claim is of course tempered with an admission of our lack of understanding of factors promoting postoperative inflammation, keeping in mind that there is evidence that preservatives used in such medications may play an important role in the pathogenesis of CME.

In cases where the risks of ocular pathology related to high IOP necessitate the use of prostaglandin analogs during the first few weeks of the postoperative period, the concomitant use of both topical NSAIDs and steroids is prudent. There is no good evidence that the use of adjunctive topical NSAIDs blunts the IOP- lowering effect of prostaglandin analogs. ${ }^{22-23}$ Prostaglandins, if needed, are routinely resumed at approximately one month following cataract surgery, at which time these agents can replace aqueous suppressant therapy that was initiated in the early postoperative period. This one month hiatus from prostaglandin therapy is, once again, arbitrary with a shorter or longer duration being recommended in eyes with postoperative ocular inflammation that is lesser or greater than average respectively. When pseudophakic eyes require longterm IOP-lowering therapy, however, prostaglandin analogs in most circumstances remain our preferred first-line agents after the first- postoperative month.

\section{REFERENCES}

1. Miyake K, Ibaraki N, Goto Y, et al. ESCRS Binkhorst lecture 2002: pseudophakic preservative maculopathy. J Cataract Refract Surg 2003;29:1800-10.

2. McNamara JA. Macular diseases. In: Ho A, Brown G, McNamara J, et al eds. Retina: Color Atlas and Synopsis of Clinical Ophthalmology. New York: McGraw-Hill 2003:32-34.

3. Ahad MA, McKee HDR. Stopping prostaglandin analogues in uneventful cataract surgery. J Cataract Refract Surg 2001;30:264445.

4. Wright PL, Wilkinson CP, Balyeat HD, Popham J, Reinke M. Angiographic cystoid macular edema after posterior chamber intraocular lens implantation. Arch Ophthalmol 1988;106:74044. 
5. Wand M, Gaudio AR, Shields MB. Latanoprost and cystoid macular edema in high-risk aphakic or pseudophakic eyes. J Cataract Refract Surg 2001;27:1397-1401.

6. Gass JD, Norton EW. Follow-up study of cystoid macular edema following cataract extraction. Trans Am Acad Ophthalmol Otolaryngol 1969;73:665-82.

7. Dhingra N, Morgan JE. Pseudophakic cystoid macular edema: 30 months after latanoprost challenge. Eye 2007;21:269-71.

8. Altintas O, Yuksel N, Karabas VL, Demirci G. Cystoid macular edema associated with latanoprost after uncomplicated cataract surgery. Eur J Ophthalmol 2005;15:158-61.

9. Wand M, Gaudio AR. Cystoid macular edema associated with ocular hypotensive lipids. Am J Ophthalmol 2002;133:403-05.

10. Jager M, Jonas JB. Cystoid macular edema associated with latanoprost therapy in a pseudophakic vitrectomized patient after removal of silicone oil endotamponade. Eur J Ophthalmol 2003;13:221-22.

11. Yeh PC, Ramanthan S. Latanoprost and clinically significant cystoid macular edema after uneventful phacoemulsification with intraocular lens implantation. J Cataract Refract Surg 2002;28:1814-18.

12. Moroi SE, Gottfredsdottir MS, Schteingart MT, et al. Cystoid macular edema associated with latanoprost therapy in a case series of patients with glaucoma and ocular hypertension. Ophthalmology 1999;106:1024-29.

13. Henderson BA, Kim JY, Ament CS, et al. Clinical pseudophakic cystoid macular edema. J Cataract Refract Surg 2007;33: 1550-58.

14. Arcieri ES, Santana A, Rocha FN, Guapo GL, Costa VP. Bloodaqueous barrier changes after the use of prostaglandin analogues in patients with pseudophakia and aphakia: a 6-month randomized trial. Arch Ophthalmol 2005;123:186-92.

15. Asano S, Miyake K, Ota I, et al. Reducing angiographic cystoid macular edema and blood-aqueous barrier disruption after smallincision phacoemulsification and foldable intraocular lens implantation: multicenter prospective randomized comparison of topical diclofenac $01 \%$ and betamethasone $0.1 \%$. J Cataract Refract Surg 2008;34:57-63.

16. Miyake K, Ibaraki N. Prostaglandins and cystoid macular edema. Surv Ophthalmol 2002;47(suppl 1):S203-18.
17. Nishi O, Nishi K, Imanishi M. Synthesis of interleukin-1 and prostaglandin E2 by lens epithelial cells of human cataracts. Br J Ophthalmol 1992;76:338-41.

18. Esquenazi, Salomon. Cystoid Macular Edema in a Pseudophakic Patient after Switching from Latanoprost to BAK-free Travopost. Journal of Ocular Pharmacology and Therapeutics 2007;23.

19. Kim DD, Doyle JW, Smith MF. Intraocular pressure reduction following phacoemulsification cataract extraction with posterior chamber lens implantation in glaucoma patients. Ophthalmic Surg Lasers 1999;30:37-40.

20. Issa SA, Pacheco J, Majmood U, et al. A novel index for predicting intraocular pressure reduction following cataract surgery. Br J Ophthalmol 2005;89:543-46.

21. Merkur A. Intraocular pressure decrease after phacoemulsification in patients with pseudoexfoliation syndrome. J Cataract Refract Surg 2001;27:528-32.

22. Flach AJ, Jampol LM, Weinberg D. Improvement in visual acuity in chronic aphakic and pseudophakic cystoid macular edema after treatment with topical 0.5\% ketorolac tromethamine. Am J Ophthalmol 1991;112:514-19.

23. Rho DS. Treatment of acute pseudophakic cystoid macular edema: diclofenac versus ketorolac. J Cataract Refract Surg 2003;29:2378-84.

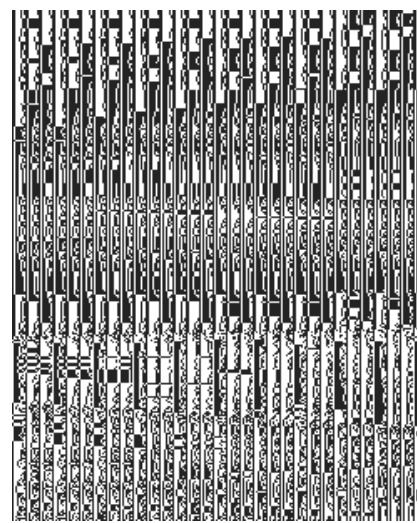

Anurag Shrivastava (anurag3@yahoo.com) 